\title{
Electric-field dependence of the hole drift mobility in molecularly doped polymers: Importance of the disorder of hopping sites
}

\author{
Yoshihiko Kanemitsu, Hiroshi Funada, and Yasuaki Masumoto \\ Institute of Physics, University of Tsukuba, Tsukuba, Ibaraki 305, Japan
}

(Received 6 May 1991; accepted for publication 27 September 1991)

\begin{abstract}
The electric-field dependence of the drift mobility of holes in a polymer matrix doped with various different molecules was studied by means of time-of-flight (TOF) photoconductivity measurements. It was first found that the degree of the positive field dependence of the drift mobility increases with decreasing magnitude of the drift mobility at zero field. The tail of the Gaussian TOF current signal becomes broad in molecularly doped polymers having a large disorder of hopping site energies. It was pointed out that the field dependence of the mobility and the energy barrier for charge transport are largely related to energetic disorder of hopping sites.
\end{abstract}

\section{INTRODUCTION}

Dynamics of charge-carrier transport in amorphous organic photoconductors has been the subject of numerous investigations. ${ }^{1-14}$ One class of amorphous organic photoconductors, molecularly doped polymers (MDPs), is a solid solution of a charge-transporting molecule in an inert polymer matrix. These MDPs become particularly attractive because of their importance as photoreceptors in electrophotography and the construction of transport theories in amorphous solids. ${ }^{2-14}$ It has been recognized that charge transport in MDPs occurs by hopping between doped molecules. However, the microscopic details of the hopping process occurring in MDPs is not well understood. Two models are currently discussed: whether polaron formation $^{7,9}$ or disorder ${ }^{8,12}$ dominates charge transport in MDPs.

It is known that the drift mobility of holes in MDPs strongly depends on the applied electric field $E$. Many experimental observations ${ }^{13}$ show the field dependence of the hole drift mobility is described as $\exp \left(E^{1 / 2}\right)$. It was theoretically pointed out that the large field dependence of mobility is attributable to disorder of hopping sites. ${ }^{8}$ The field and temperature dependence of the drift mobility in a polymer matrix doped with a molecule can be explained by the disorder picture proposed by Bässler and his co-workers. ${ }^{8,12}$ However, the origin of disorder of hopping sites is not clear. On the other hand, the polaron model rests on the idea that the geometry and energy of doped molecules in neutral and cation forms are different. Hole transport involves molecular distortion which depends on the chemical structure of doped molecules. Therefore, studies of the field dependence of the drift mobility in various different systems would reveal the effects of polaron formation and disorder on dynamics of hopping charge transport in MDPs. In this work, we have studied the electric-field dependence of the drift mobility of holes in a polycarbonate matrix doped with various different molecules by means of time-of-flight (TOF) photoconductivity measurements.

\section{EXPERIMENTAL PROCEDURES}

Hole-transporting molecules used in this work were OXD [2,5-bis(4'-diethylaminophenyl)-1,3,4-oxadiazole], PRA [1-phenyl-3-( $p$-diethylaminostyryl)-5-( $p$-diethylaminophenyl)-2-pyrazoline], DEH ( $p$-diethylaminobenzaldehyde-1,1'-diphenylhydrazone), HD (1-phenyl-1,2,3, 4-tetrahydroquinoline-6-carboxyaldehyde-1,1'-diphenylhydrazone), BD [1,1-bis(4-diethylaminophenyl)-4,4-diphenyl-1,3-butadiene], PS (4- $N, N$-diphenylamino- $\alpha$-phenylstilbene), and MPS [4- $N, N$-bis (4-methylphenyl)amino- $\alpha$ phenylstilbene]. These hole-transporting molecules were dispersed in bisphenol- $A$-polycarbonate. Since the ionization potential of polycarbonate is larger compared with that of hole-transporting molecules, ${ }^{14}$ the polycarbonate is an ideal matrix. These in methylene chloride were coated onto an aluminized Mylar sheet. The dry film thickness was $12 \mu \mathrm{m}$. The concentration of molecules in solid polycarbonate films was $50 \mathrm{wt}$ \%. A semitransparent gold electrode was finally deposited by vacuum evaporation. In the TOF photoconductivity experiment, the sample was connected in a circuit containing a voltage source and a resistance. The MDPs films were excited through the gold electrode by a $0.3-\mathrm{ns}, 337-\mathrm{nm}$ pulse from a $\mathrm{N}_{2}$ laser. In some experiments, the $0.2-\mu \mathrm{m}$ charge generation films of metal-free phthalocyanine or amorphous selenium were attached to MDPs films. These two-layer structures were excited by a $0.5-\mu \mathrm{s}, 600-\mathrm{nm}$ pulse from a xenon flash lamp. The current transients were displayed on a digital storage oscilloscope interfaced to a computer.

\section{RESULTS AND DISCUSSION}

Figure $l$ shows typical photocurrent pulse shapes. In all sample films, TOF signals show well-developed plateau regions suggestive of nondispersive transport. The transit time $t_{T}$ was determined by the shoulder in the TOF shape, ${ }^{11}$ and the arrows in Fig. 1 indicate the transit times. The hole drift mobility $\mu$ is given by $\mu=L^{2} / t_{T} V$, where $L$ is the sample thickness and $V$ is the applied voltage. Figure 2 (a) summarizes the field dependence of the drift mobility of a polymer matrix doped with various different mole- 

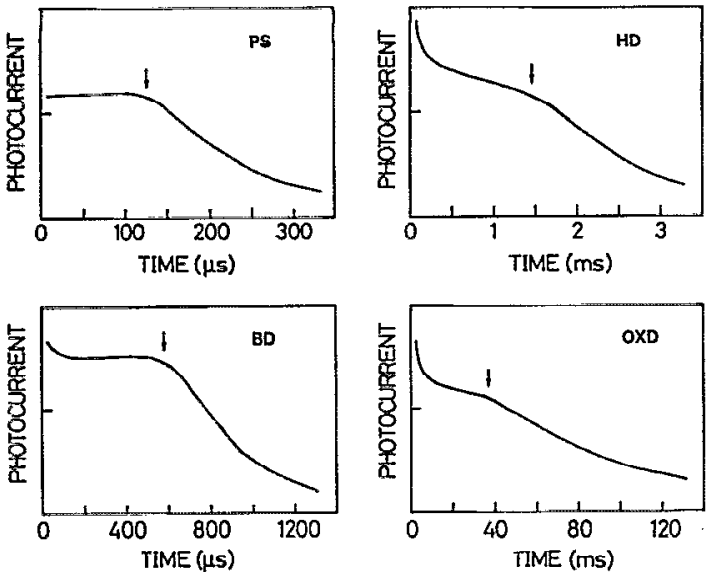

FIG. 1. Typical TOF photocurrent pulse shapes measured at $2.0 \times 10^{5}$ $\mathrm{V} / \mathrm{cm}$ and $298 \mathrm{~K}$. The arrows indicate the transit times.

cules. We note a relation of $\mu \propto \exp \left(E^{1 / 2}\right)$ in various MDPs. Moreover, Fig. 2(b) shows the field dependence of the drift mobility of holes in PS and OXD mixed doped polymers. The total concentration of PS and OXD molecules in a polymer matrix was $50 \mathrm{wt}$. \%, and the relative concentration of OXD to PS was changed. There is no ionization potential difference between OXD and PS molecules. ${ }^{14}$ Even in PS and OXD mixed doped systems, the mobility also shows the $\exp \left(E^{1 / 2}\right)$ dependence. The slopes in Fig. 2 give the degree of the field dependence, $S=\partial \ln \mu / \partial E^{1 / 2}$. It is seen from Fig. 2 that $S$ decreases with increasing magnitude of the drift mobility at zero field, $\mu(E=0)$.

In Fig. 3, the logarithm of $\mu(E=0)$ is plotted as a

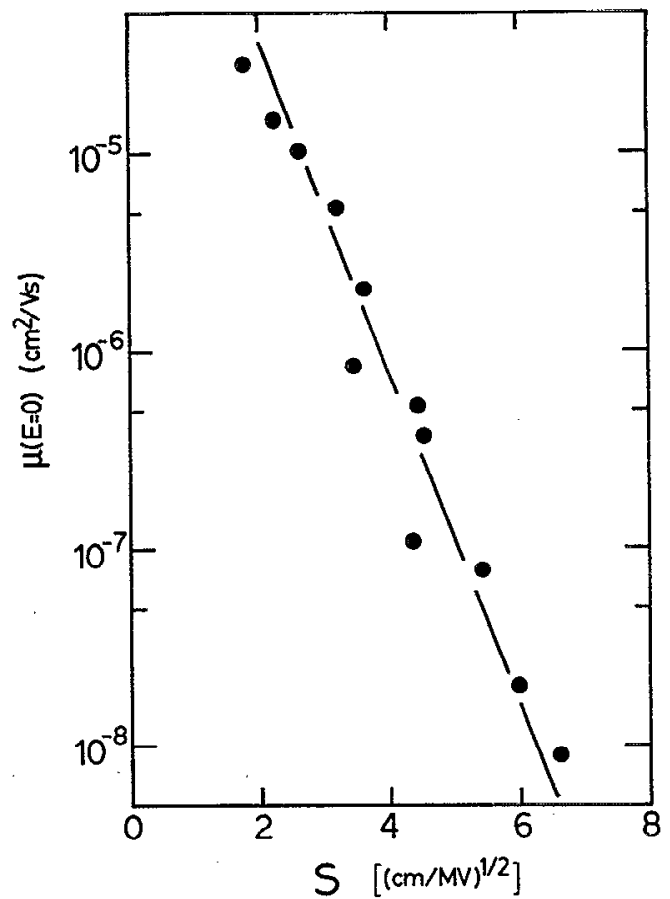

FIG. 3. The drift mobility of holes at zero field, $\mu(E=0)$, as a function of the field dependence $S, S=\partial \ln \mu / \partial E^{\mathrm{t} / 2}$.

function of $S$. We first found a phenomenological relation expressed as

$$
\mu(E=0) \propto \exp (-S) .
$$

The mobility data collected for chemically quite different molecules and mixed doped systems suggest that the field dependence of the mobility is determined by a universally
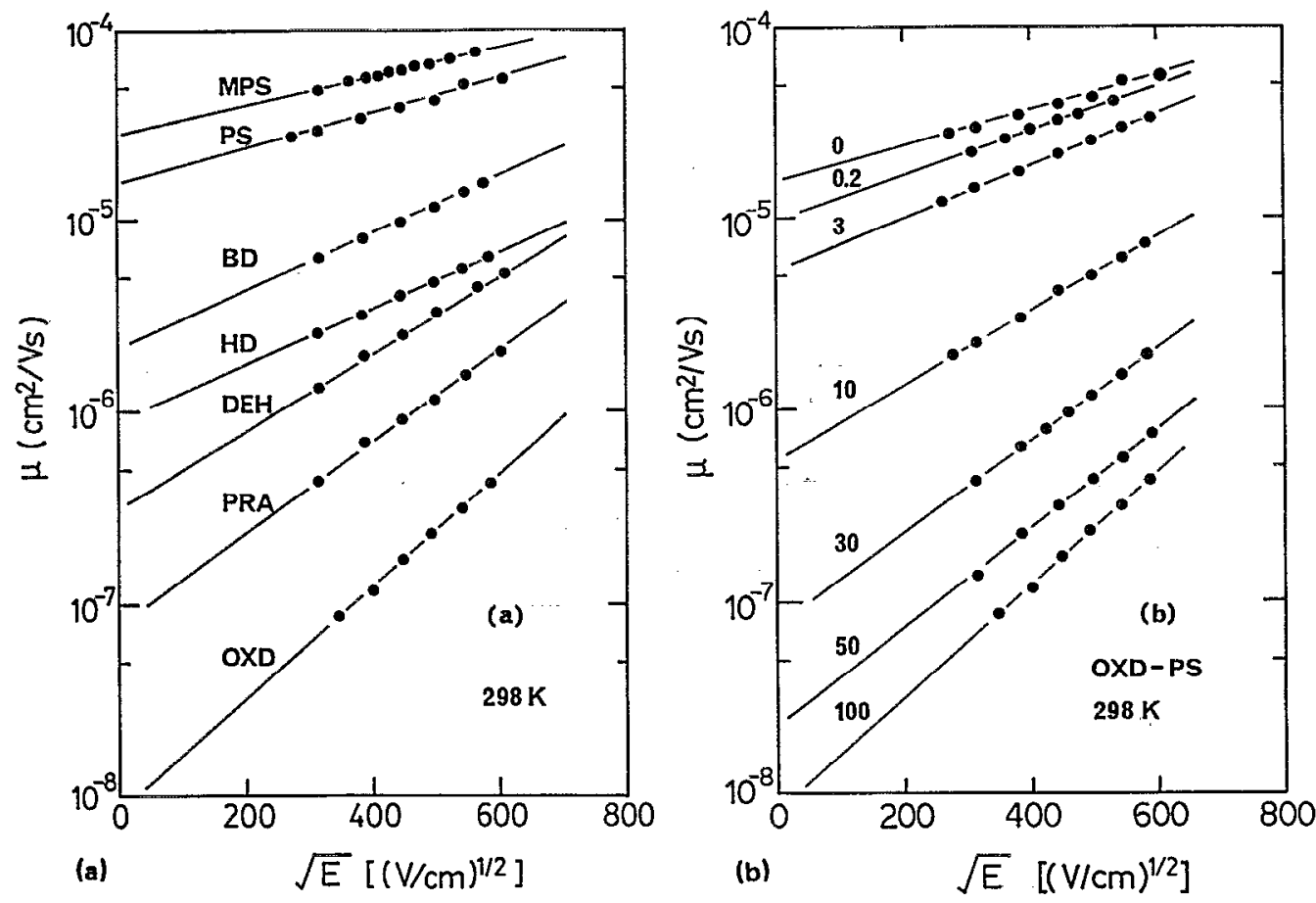

FIG. 2. (a) The electric-field dependence of the drift mobility of holes in a polymer matrix doped with various different molecules at $298 \mathrm{~K}$. (b) The electric-field dependence of the hole drift mobility in mixed doped PS and OXD systems at $298 \mathrm{~K}$. The numbers mean the relative concentration (wt \%) of OXD to PS molecules. 


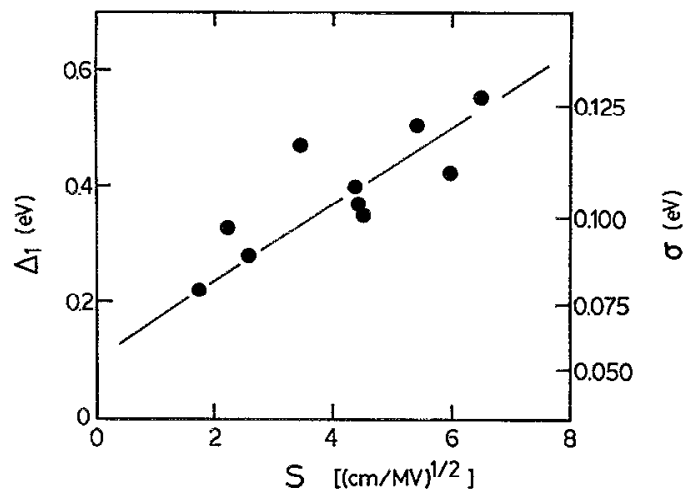

FIG. 4. The activation energy of the hole mobility at $2.0 \times 10^{5} \mathrm{~V} / \mathrm{cm}, \Delta_{1}$, and energetic disorder of hopping sites $\sigma$, as a function of the field dependence $S$.

physical origin in amorphous MDPs rather than particular structures and distortions of doped molecules.

The temperature dependence of the drift mobility of holes is usually given by the Arrhenius law: $\mu \propto \exp \left(-\Delta_{1} / k T\right)$. In the polaron picture, hole transport is an activation process and the temperature dependence of the mobility is determined by the Arrhenius law. On the other hand, Bässler et al. ${ }^{2}$ predicted that the temperature dependence of the mobility is given by $\mu \propto \exp \left[-\left(\Delta_{2} / k^{4} T\right)^{2}\right]$, where $\Delta_{2}$ is related to the energy width of the distribution of hopping sites, $\sigma$. However, we cannot clearly distinguish whether $T^{-1}$ or $T^{-2}$ is a better description. Hereafter, we used $\Delta_{1}$ as the overall activation energy for charge transport and $\sigma$ as the degree of the energetic disorder of hopping sites. Figure 4 shows $\Delta_{1}$ at $2.0 \times 10^{5} \mathrm{~V} / \mathrm{cm}$ and $\sigma$ at $298 \mathrm{~K}$ as a function of $S$ at $298 \mathrm{~K}$. Since $S$ increases with increasing $\sigma, S$ is a good parameter reflecting the degree of disorder of hopping sites.

If the energy difference between the nearest-neighbor hopping sites is very small compared with the polaron binding energy, $\Delta_{1}$ in the polaron picture is given by $\Delta_{1}=E_{p} / 2-J(r)$, where $E_{p}$ is the polaron binding energy and $J(r)$ is the overlap integral between hopping sites. At high molecular concentrations, the activation energy in many MDPs depends on the molecular concentration. ${ }^{3,9,10}$ The charge transport may be due to the adiabatic small polaron motion and $J(r)$ may be large enough to affect the value of $\Delta_{1}{ }^{9}$ Positional disorder also affects the value of $\Delta_{1}$ through the fluctuation of $J(r)$. If there is no energetic disorder of hopping sites, the field-induced localization of carriers decreases the mobility, and the field dependence of the mobility becomes negative. ${ }^{8}$ Therefore, the positive field dependence $S$ shows that disorder part of the hopping barrier is not negligibly small compared with the polaron binding energy. Moreover, Fig. 4 shows that $\Delta_{1}$ increases with increasing $\sigma$ : The hopping barrier is strongly influenced by disorder of hopping sites. The overall "energy barrier for hopping consists of disorder and polaron parts. In particular, we consider that in MDPs having low hole mobility, energetic disorder determines charge transport. In very low-mobility regions, the TOF signal becomes

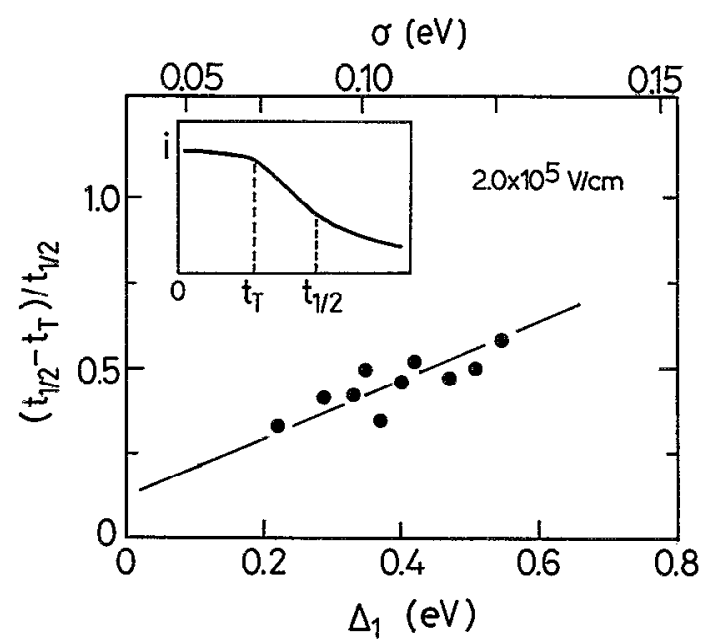

FIG. 5. The tail broadening of the Gaussian TOF signal, $\left(t_{1 / 2}-t_{T}\right) / t_{1 / 2}$, vs energetic disorder of hopping sites $\sigma$ and the activation energy $\Delta_{1}$. The inset illustrates a typical TOF signal. $t_{T}$ is the time at which the current initially drops and $t_{1 / 2}$ is the time at which the current drops to one-half of the value at $t_{T}$.

dispersive $\mathrm{e}^{11,15}$ and trap states and impurities in MDPs are considered to control charge transport. Trap states and impurities also affect the energetic distribution of hopping sites and hopping barrier.

Moreover, the observed TOF signal is characterized by the tail broadening. As the TOF signal is asymmetrical, it is useful to adopt the quantity $\left(t_{1 / 2}-t_{T}\right) / t_{1 / 2}$ as the degree of dispersion. ${ }^{5}$ Here $t_{1 / 2}$ is the time when the photocurrent drops to one-half of the value at $t_{T}$. In Fig. 5, $\left(t_{1 / 2}-t_{T}\right) / t_{1 / 2}$ is plotted as a function of energetic disorder $\sigma$ and the activation energy $\Delta_{1}$. The dispersion, $\left(t_{1 / 2}-t_{T}\right) / t_{1 / 2}$, increases with increasing $\sigma$ and $\Delta_{1}$. This fact suggests that the nondispersive Gaussian TOF signal may be observed in a MDP having a small energy fluctuation of hopping sites. By using Monte Carlo simulation techniques, Pautmeier and co-workers ${ }^{8,12}$ showed that the tail broadening of the Gaussian TOF signal is caused by energetic disorder of hopping sites. By comparing our experimental results with their simulations, it is concluded that the hopping barriers are largely rclated to energetic disorder to hopping sites. Energetic disorder of hopping sites dominates hopping charge transport in MDPs, and consequently we can observe the simple relation of $\mu(E$ $=0) \propto \exp (-S)$ in various different systems. However, at present, the origin of disorder of hopping sites in MDPs of binary solid solution is not clear.

The structural and energetic consideration of doped molecules help to provide the microscopic understanding of disorder and dynamics of charge transport in MDPs. Figures 3 and 4 show that $\sigma$ decreases with increasing $\mu(E=0)$. In high-mobility MDPs, disorder of hopping sites decreases and the intramolecular process plays an important role in charge transport. Using a molecular orbital calculation technique (MOPAC), Sugimoto et al. ${ }^{16}$ studied the stable geometries of molecules in neutral and cation forms and the energetic difference between the neutral and 
cation states, $\Delta E_{C-N}$. The calculated values of $\Delta E_{C-N}$ were $0.55 \mathrm{eV}$ for MPS, $0.68 \mathrm{eV}$ for PS, $0.81 \mathrm{eV}$ for $\mathrm{HD}$, and $0.83 \mathrm{eV}$ for DEH. Since hole transport is a chain of the redox process between neutral and cation states, $\Delta E_{C-N}$ contributes to the overall activation energy for charge transport and large $\Delta E_{C-N}$ causes low hole mobility. In the polycarbonate doped with MPS, PS, HD, and DEH molecules, there is a close relationship between $\mu(E=0)$ and $\Delta E_{C-N}$ : The magnitude of the mobility $\mu(E=0)$ [see Fig. 2(a)] is inversely proportional to the value of $\Delta E_{C-N}$. Therefore, we consider that in high-mobility MDPs, the intramolecular process of doped molecules plays an important role in charge transport in binary solid solutions. It is expected that the geometries and structures of doped molecules and polymer matrix during hole transport studied by molecular orbital calculations provide the detailed understanding of the dynamics of charge transport and the nature of disorder of hopping sites in MDPs.

\section{CONCLUSION}

In conclusion, we have studied TOF photocurrent pulse shapes and the electric-field dependence of the mobility in a polymer matrix doped with various different molecules. The field dependence of the mobility and the overall energy barrier for charge transport are largely related to the degree of energetic disorder of hopping sites.
Finally, it was pointed out that the intramolecular process plays a dominant role in charge transport in MDPs having high mobility.

\section{ACKNOWLEDGMENTS}

The author would like to thank Y. Sugimoto of Hitachi Chemical Co., Ltd. for computer calculations of molecular orbitals. This work was supported in part by the CIBAGEIGY foundation for the Promotion of Science.

'J. Mort and G. Pfister, Electronic Properties of Polymers, edited by J. Mort and G. Pfister (Wiley, New York, 1982), p. 215.

${ }^{2}$ H. Bässler, G. Schönherr, M. Abkowitz, and D. M. Pai, Phys. Rev. B 26, 3105 (1982).

${ }^{3}$ M. Stolka, J. F. Yanus, and D. M. Pai, J. Phys. Chem. 88, 4707 (1984).

${ }^{4}$ J. S. Facci and M. Stolka, Philos. Mag. B 54, 1, (1986).

${ }^{5}$ H. J. Yuh and M. Stolka, Philos. Mag. B 58, 539 (1988).

${ }^{6}$ A. Peled and L. B. Schein, Chem. Phys. Lett. 153, 422 (1988).

${ }^{7}$ J. X. Mack, L. B. Schein, and A. Peled, Phys. Rev. B 39, 7500 (1989).

${ }^{8}$ L. Pautmeier, R. Richert, and H. Bässler, Synth. Met. 37, 271 (1990).

${ }^{9}$ L. B. Schein, D. Glatz, and J. C. Scott, Phys. Rev. Lett. 65, 472 (1990).

${ }^{10}$ P. M. Borsenberger, J. Appl. Phys. 68, 5188 (1990); 68, 5682 (1990).

${ }^{11}$ Y. Kanemitsu and J. Einami, Appl. Phys. Lett. 57, 673 (1990).

${ }^{12}$ L. Pautmeier, R. Richert, and H. Bässler, Philos. Mag. B 63, 587 (1991).

${ }^{13}$ See, for example, L. B. Schein, A. Peled, and D. Glatz, J. Appl. Phys. 66, 686 (1989).

${ }^{14}$ Y. Kanemitsu, H. Funada, and Y. Masumoto, Appl. Phys. Lett. 59, 697 (1991).

${ }^{15}$ Y. Kanemitsu and D. Imanishi, J. Appl. Phys. 66, 4526 (1989).

${ }^{16}$ Y. Sugimoto, S. Hayashida, and Y. Kanemitsu (unpublished). 